\title{
DIPHASIASTRUM THYOIDES (LyCOPODIACEAE), NUEVO REGISTRO PARA LA FLORA URUGUAYA
}

\author{
H. ANDRES GONZALEZ1', CARLOS A. BRUSSA ${ }^{1,2}$ y MARCELO D. ARANA ${ }^{3}$
}

\begin{abstract}
Summary: Diphasiastrum thyoides (Lycopodiaceae), new record for the flora of Uruguay. Diphasiastrum thyoides (Lycopodiaceae) is registered for first time for the flora of Uruguay. It was collected in the departments of Maldonado and Cerro Largo. The new record extends the distribution to the south, establishing the southern Uruguay as its southernmost distributional limit of the species. A description, a distribution map in the country, as well as pictures of the species and an identification key for all Uruguayan Lycopodiaceae are provided.
\end{abstract}

Key words: Diphasiastrum, Lycopodiidae, Maldonado, Cerro Largo, Uruguay.

Resumen: Se registra por primera vez para Uruguay la especie neotropical Diphasiastrum thyoides (Lycopodiaceae), colectada en los departamentos de Maldonado y Cerro Largo. El registro extiende su área geográfica hacia el sur, estableciendo el sur de Uruguay como el límite austral de distribución de la especie. Se brinda una descripción, un mapa de distribución en el país, así como ilustraciones con fotografías de la especie y una clave para la identificación de todas las Lycopodiaceae uruguayas.

Palabras clave: Diphasiastrum, Lycopodiidae, Maldonado, Cerro Largo, Uruguay.

\section{INTRODUCCIÓN}

Diphasiastrum Holub es un género de Lycopodiaceae que cuenta con unas 22 especies distribuidas ampliamente en América, Europa y Asia en sitios de alta humedad o con presencia de nieve en la época invernal (Wagner \& Beitel, 1993). Las especies se caracterizan por ser plantas terrestres o rupícolas, con tallos principales subterráneos o epígeos, reptantes, cilíndricos, isofilos, desde los cuales emergen raíces de su cara ventral. Las ramas laterales son erectas, cilíndricas en la base y dorsiventralmente aplanadas hacia el ápice, anisofilos en las especies sudamericanas, con

1 Facultad de Agronomía, Universidad de la República, Avda. E. Garzón 780, Montevideo, Uruguay, CP 12.900, Email: andres260390@gmail.com

2 Museo y Jardín Botánico de Montevideo "Prof. A. Lombardo", 19 de Abril 1181, Montevideo, Uruguay, CP 11.700 .

3 Depto. Ciencias Naturales, Facultad de Ciencias Exactas, Físico-Químicas y Naturales Universidad Nacional de Río Cuarto, Ruta 36 km 601, X5804ZAB, Río Cuarto, Córdoba, Argentina los licofilos dispuestos en 4 series, dos laterales, de licofilos anchos, una dorsal y una ventral de licofilos más angostos casi filiformes. Estos sistemas de ramas laterales presentan ramificación anisotómica. Los estróbilos se disponen sobre pedúnculos erectos ramificados o sésiles (en una especie no sudamericana), los esporofilos son subpeltados más cortos que los licofilos del pedúnculo. Los esporangios son reniformes y las esporas son reticuladas en todas las caras con lados y ecuador convexos y ángulos agudos (Holub, 1975; Arana \& Øllgaard, 2012).

En Sudamérica habitan dos especies del género, de las cuales Diphasiastrum thyoides (Humb. \& Bonpl. ex Willd.) Holub, presenta una amplia distribución desde México y Guatemala en Mesoamérica hasta los países del Cono sur de Sudamérica, Argentina, Bolivia y Brasil (Mickel \& Smith, 2004; Arana \& Øllgaard, 2012; Øllgaard \& Windisch, 2014).

Durante diversos viajes de recolección, en el marco de la actualización florística de Uruguay, se colectaron ejemplares de Diphasiastrum thyoides, especie que no estaba registrada para la flora 
uruguaya; por lo que en el presente trabajo se cita por primera vez la mencionada especie para Uruguay, se describe e ilustra mediante fotografías y se incluye una clave para todas las entidades de la familia Lycopodiaceae presentes en el país. Esta cita constituye una novedad a nivel genérico para la flora uruguaya, complementando los géneros de Lycopodiaceae Lycopodiella Holub, Palhinhaea Vasc. \& Franco y Pseudolycopodiella Holub, citados previamente (Legrand \& Lombardo, 1958; Arana \& Øllgaard, 2012).

\section{Material y Método}

El material fue coleccionado durante viajes de relevamiento de flora en las Sierras de Valdivia al norte del departamento de Maldonado y en el departamento de Cerro Largo. Los ejemplares colectados fueron determinados con la ayuda de bibliografía específica, el estudio de imágenes de los ejemplares tipo y el análisis de caracteres morfológicos de ejemplares depositados en los herbarios BA, COL, CORD, LIL, MA, P, RCV y SI. El material de referencia fue depositado en los herbarios MVJB y MVFA.

\section{Resultados}

Diphasiastrum thyoides (Humb. \& Bonpl. ex Willd.) Holub, Preslia 47 (2): 108. 1975. Fig. 1 A, B, C y D.

=Lycopodium thyoides Humb. \& Bonpl. ex Willd., Sp. Pl. 5: 18. 1810. Lycopodium complanatum L. var thyoides (Willd.) Christ, in Schwacke, Pl. Nov. Min. 42. 1900. Tipo: Venezuela, Silla de Caracas, A. von Humboldt s.n. (holotipo B-W 19352!).

=Lycopodium comptonioides Desv. Mém. Soc. Linn. Paris. 6: 185. 1827. (excl.. sinonimos). Tipo: Brasil, locis montosis Brasiliæ, Herbier A. N. Desvaux s.c, s.n. (holotipo P00559221!).

Ilustraciones: de la Sota (1977: 24, fig. 5 H-O); Mickel \& Beitel (1988: fig. 2A); Nessel (1955: fig. 107); Øllgaard (1988: fig. 25A).

Plantas terrestres; tallos principales cilíndricos rastreros de $1 \mathrm{~cm}$ de diámetro, radicantes en la base. Licofilos pequeños; lineal-subulados, isomorfos de $3-4 \times 0,3-0,4 \mathrm{~mm}$. Tallos principales erectos o algo decumbentes de $30-45 \mathrm{~cm}$ de altura, con licofilos, lineal-subulados, isomorfos de $2-3 \times$ 0,3-0,5 $\mathrm{mm}$, dispuestos helicoidalmente; tallos laterales de ramificación dicotómica, aplanados dorsiventralmente. Licofilos adpresos, base soldada al eje caulinar y ápice libre, acuminados, dispuestos en cuatro series: dos laterales, con licofilos claramente desarrollados, deltoides, de base largamente decurrente y ápice agudo a acuminado, adpresos, de $3-5 \times 0,5-1 \mathrm{~mm}$; uno dorsal subulado, adpreso, base conspicuamente decurrente de $3-5 \times 0,5-0,8 \mathrm{~mm}$ y uno ventral inconspicuo, acicular a filiforme, sin base decurrente de $1-1,5 \times 0,3-0,5 \mathrm{~mm}$. Tallos fértiles que nacen lateralmente de la parte basal de los ejes erectos, ramificados en el ápice dicotómicamente, de 15-20 cm de largo, licofilos basales muy próximos, los superiores distantes, dispuestos helicoidalmente de 2,5-3 $\times 0,3-0,6 \mathrm{~mm}$. Estróbilos 4-8 por tallo fértil de $20-40 \times 2,5-3,3 \mathrm{~mm}$. Esporofilos subpeltados, deltoides, acuminados, dispuestos helicoidalmente, con márgenes escariosos, de 2-2,5 × 1,3-1,5 mm. Esporas globoso-tetraédricas, anisopolares, contorno circular, con ornamentación muriforme. Diámetro ecuatorial de (35) 35(37) $\pm 1,20 \mu \mathrm{m}$, reticuladas en la cara distal y proximal. Retículo distal regular, con aréolas amplias y grandes y superficie con microgránulos y micropliegues, retículo proximal irregular, superficie de la cara proximal con microgránulos. Lesura crestada con labios angostos definidos, sin llegar hasta el ecuador.

Distribución y hábitat. Ésta especie presenta una amplia distribución desde México y Guatemala hasta las provincias del noroeste y centro de la Argentina, estando presente en las provincias de Jujuy, Salta y Córdoba en la Argentina (Arana \& Øllgaard, 2012), así como en los estados de Pará, Piauí, Minas Gerais, Rio de Janeiro, São Paulo, Paraná, Santa Catarina, and Rio Grande do Sul (Øllgaard \& Windisch, 2014). En Uruguay se la ha encontrado en las cercanías de la localidad de Arévalo en Cerro Largo y Sierras de Valdivia al norte del departamento de Maldonado, punto que constituye el límite austral de su distribución (Fig. 2). En Uruguay es epipétrico y habita en paredones poco húmedos a pleno sol, ocupando un estrato horizontal de arenisca a unos 2 metros de altura sobre el suelo y tapizando el sotobosque en una superficie no mayor a los $50 \mathrm{~m}^{2}$. 


\section{H.A. González et al. - Diphasiastrum thyoides en la flora uruguaya}
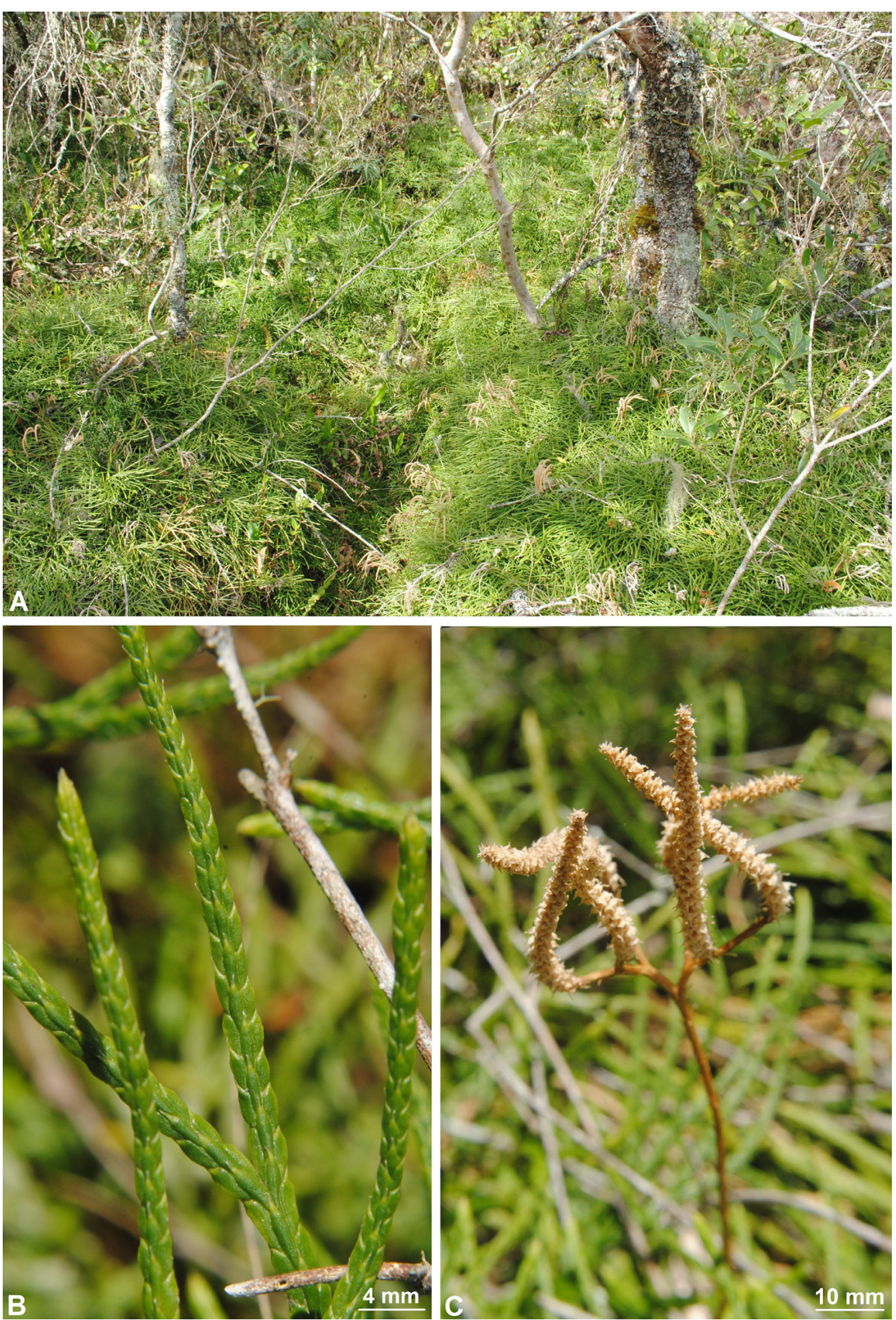

Fig. 1. Diphasiastrum thyoides. A: En su hábitat tapizando el sotobosque en Sierras de Valdivia, Maldonado, Uruguay. B: Detalle de ramas laterales donde se pueden observar los licofilos laterales y el licofilo dorsal. C: Detalle de eje fértil con estróbilos maduros. 


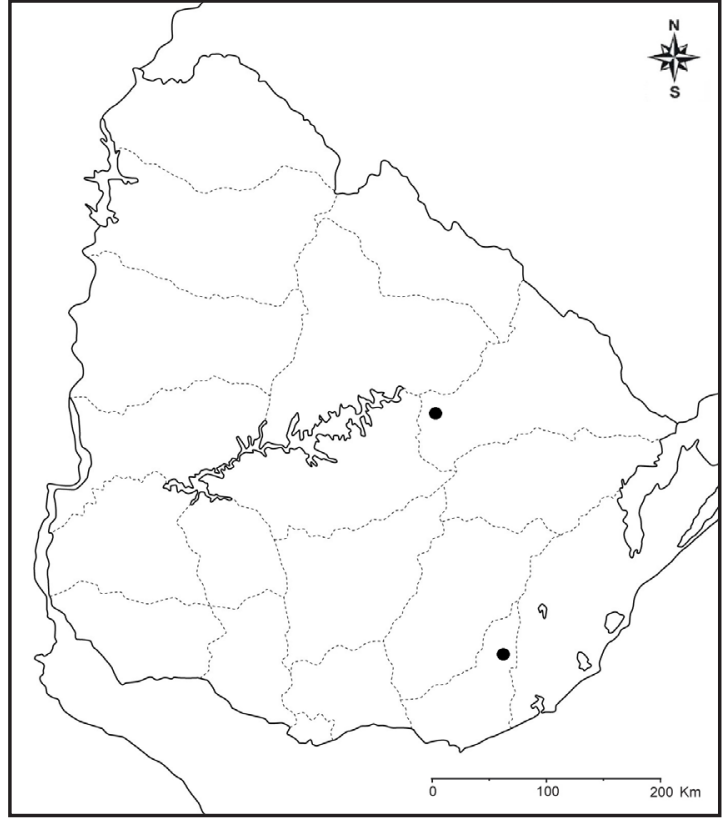

Fig. 2. Mapa de distribución geográfica de Diphasiastrum thyoides en Uruguay.
Fenología. Diphasiastrum thyoides produce los estróbilos en noviembre y diciembre y los mismos pueden encontrarse maduros sobre las plantas durante un largo período de tiempo.

Observaciones. Según Wilce (1965) y Arana \& Øllgaard (2012), ésta especie es variable y su morfología se correlaciona en alto grado con los factores ambientales; muy probablemente sea un complejo de especies que necesita un mayor estudio (Wilce, 1965; Øllgaard, 1994). Se diferencia de Diphasiastrum falcatum B. Øllg. \& P.G.Windisch, la otra especie sudamericana del género y que habita en Brasil, ya que esta última posee los licofilos laterales con el ápice fuertemente falcado.

Material examinado. URUGUAY. Depto. Cerro

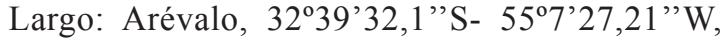
8-XI-2013, Brussa, s.n. (MVJB 28743). Depto. Maldonado: Sierras de Valdivia, 3416'11'S543ㅇ'36"W, 13-X-2013, Aldabe, Abreu \& González 1039 (MVFA).

\section{Clave para determinar las especies de la familia Lycopodiaceae presentes en Uruguay.}

1. Estróbilos péndulos terminales sobre ramas laterales horizontales.. Palhinhaea cernua (L.) Vasc. \& Franco

1 '. Estróbilos erectos, terminales, sobre tallos erectos simples o bifurcados.

2. Licofilos dispuestos en cuatro hileras, una dorsal y una ventral de licofilos angostos y dos laterales de licofilos más anchos, estróbilos sobre pedúnculos con licofilos muy reducidos y espaciados, esporas reticuladas Diphasiastrum thyoides

2'. Licofilos de filotaxia subverticilada o dispuestos en tres hileras, una dorsal de licofilos cortos, angostos y dos laterales de licofilos anchos y largos, estróbilos sobre pedúnculos con licofilos no reducidos, aproximados, esporas ruguladas.

3. Tallos horizontales anisofilos, con licofilos laterales más largos que los dorsales, esporangios isovalvados. Pseudolycopodiella meridionalis (Underw. \& F. E. Lloyd) Holub

3'. Tallos horizontales isofilos, con licofilos iguales, subverticilados, esporangios anisovalvados.

4. Estróbilos con esporofilos patentes, no caudados, licofilos de los ejes rastreros con margen ciliado a dentado, raro entero. Lycopodiella longipes (Hook. \& Grev.) Holub

4 '. Estróbilos con esporofilos adpresos, caudados, licofilos de los ejes rastreros con margen entero a levemente denticulado. Lycopodiella tupiana (B. Øllg. \& P.G. Windisch) B. Øllg.

\section{Agradecimientos}

Los autores agradecen a Irina Aldabe y Martin Abreu por su colaboración en los viajes de colecta, al Dr. Mauricio Bonifacino por las correcciones realizadas al artículo y a los integrantes de la
Cátedra de Botánica de la Facultad de Agronomía de la Universidad de la República, por su ayuda constante, así como al editor y los comentarios de dos revisores anónimos que contribuyeron a mejorar sensiblemente el manuscrito. 


\section{Bibliografía}

ARANA, M. \& B. ØLLGAARD, 2012. Revisión de las Lycopodiaceae (Embryopsida, Lycopodiidae) de Argentina y Uruguay. Darwiniana 50(2): 266-295.

DE LA SOTA, E. R. 1977. Pteridófitas. Flora de la Provincia de Jujuy, Colección Científica del Instituto Nacional de Tecnología Agropecuaria 8: XIII+1275.

HOLUB, J. 1975. Diphasiastrum, a new genus in Lycopodiaceae. Preslia 47: 97-110.

LEGRAND, C. M. D. E. \& A. LOMBARDO. 1958. Flora del Uruguay I. Pteridophyta, pp 1-67. Montevideo: Museo Nacional de Historia Natural.

MICKEL, J. T. \& J. M. BEITEL. 1988. Pteridophyte flora of Oaxaca, Mexico. Memoirs of the New York Botanical Garden 46: 1-568.

MICKEL, J. T. \& A. R. SMITH. 2004. The pteridophytes of Mexico. Memoirs of the New York Botanical Garden 88: 1-1054.

NESSEL, H. 1955. Lycopodiaceae. In: Hoehne, F.C. Flora Brasílica II (fasc. 11): 1-131.
ØLLGAARD, B. 1988. Lycopodiaceae. In: Harling, G. \& Andersson, L. Flora of Ecuador 33: 1-155.

ØLLGAARD, B. 1994. Lycopodiaceae, en R. M. Tryon \& R. G. Stolze (eds.), Pteridophyta of Peru, Parte VI. Fieldiana, Botany, new series 34: 16-66.

ØLLGAARD, B. \& P. G. WINDISCH. 2014. Lycopodiaceae in Brazil. Conspectus of the family I. The genera Lycopodium, Austrolycopodium, Diphasium, and Diphasiastrum. Rodriguésia 65(2): 293-309.

WAGNER, W. H. JR. \& J. M. BEITEL. 1993. Lycopodiaceae, en Flora of North America. Editorial Committee (ed.), Flora of North America 1, pp 18-37. New York: Oxford University Press.

WILCE. J.H. 1965. Section Complanata of the genus Lycopodium. Beihefte Nova Hedwigia 19: i-ix, $1-233+40$ plates.

Recibido el 21 de febrero de 2015, aceptado el 23 de abril de 2015. 
